“드 2013 IEEE. Personal use of this material is permitted. Permission from IEEE must be obtained for all other uses, in any current or future media, including reprinting/republishing this material for advertising or promotional purposes, creating new collective works, for resale or redistribution to servers or lists, or reuse of any copyrighted component of this work in other works." 


\title{
Analyzing EEG Signals under Insulin-induced Hypoglycemia in Type 1 Diabetes Patients
}

\author{
Lien B. Nguyen, Anh V. Nguyen, Sai Ho Ling, Senior Member, IEEE and Hung T. Nguyen, \\ Senior Member, IEEE
}

\begin{abstract}
Hypoglycemia is dangerous and considered as a limiting factor of the glycemic control therapy for patients with type 1 diabetes mellitus (T1DM). Nocturnal hypoglycemia is especially feared because early warning symptoms are unclear during sleep so an episode of hypoglycemia may lead to a fatal effect on patients. The main objective of this paper is to explore the correlation between hypoglycemia and electroencephalography (EEG) signals. To do this, the EEG of five T1DM adolescents from an overnight insulin-induced study is analyzed by spectral analysis to extract four different parameters. We aim to explore the response of these parameters during the clamp study which includes three main phases of normal, hypoglycemia and recovery. We also look at data at the blood glucose level (BGL) of 3.3-3.9 mmol/l to find a threshold to distinguish between non-hypoglycemia and hypoglycemia states. The results show that extracted EEG parameters are highly correlated with patients' conditions during the study. It is also shown that at the BGL of 3.3 $\mathrm{mmol} / \mathrm{l}$, responses to hypoglycemia in EEG signals start to significantly occur.
\end{abstract}

\section{INTRODUCTION}

Hypoglycemia, or abnormally low blood glucose level (BGL), is the most common but dangerous complication of the intensive insulin therapy for patients with type 1 diabetes mellitus (T1DM). Hypoglycemia impacts life quality of all T1DM patients, limits their intellectual as well as physical activities, and potentially causes to irreversible severe effects, such as cognitive impairments, seizures, coma, and even death. A study in 2004 reported that severe hypoglycemia (defined as episodes in which patients need assistance to reestablish the normal BGL) happens in one third of 1076 selfreported participants with an incidence rate of 1.3 episodes/patient-year [1]. Nocturnal hypoglycemia is especially dangerous because sleep reduces and obscures early warning symptoms, so that an initially mild episode may become severe. It was reported previously that almost $50 \%$ of all episodes of severe hypoglycemia occur at night during sleep [2]. Because of its severity, intensive research has been devoted to the development of systems that can detect the onset of hypoglycemia episodes, and then give an alarm to provide enough time for patients and carers to take action.

Lien B. Nguyen, Anh V. Nguyen, Sai Ho Ling and Hung T. Nguyen are with the Centre for Health Technologies, Faculty of Engineering and Information Technology, University of Technology Sydney, Broadway, NSW 2007, Australia, (e-mail: BichLien.Nguyen@student.uts.edu.au; Anh.Nguyen-3@student.uts.edu.au; $\quad$ Steve.Ling@uts.edu.au; Hung.Nguyen@uts.edu.au).
Under the occurrence of hypoglycemia, the human brain is one of the first affected organs. Because it cannot synthesize as well as store this primary metabolic fuel, the brain depends on a continuous supply of glucose and is vulnerable to any glucose deprivation [3]. Since the electroencephalogram (EEG) is directly related to the metabolism of brain cells, a failure of cerebral glucose supply can cause early changes in EEG signals. A number of studies have reported important traces in EEG signals induced by hypoglycemia episodes in T1DM patients [4, 5]. Recent studies also lead to acceptable results which show the potential ability of detecting hypoglycemia from EEG signals [6-8]. Nevertheless, all of these results need to be improved further in order to be applied into the real clinical environment.

In our previous works, EEG signals of five T1DM adolescent patients from a glucose clamp study were analyzed to explore the difference in EEG signals between patients' non-hypoglycemia state and hypoglycemia state. Statistical analysis yields some significant changes in EEG signals which can be used efficiently as inputs of a classification algorithm for detecting hypoglycemia $[8,9]$. In this paper, we aim to investigate the correlation between four spectral EEG parameters with patients' conditions during the whole clamp study which includes three different states of normal, hypoglycemia and recovery. We also look at the BGL area of 3.3-3.9 mmol/l (60-70 mg/dl whole blood glucose) to figure out the EEG response to the potentially early onset of hypoglycemia. This paper is organized as follows. Section II provides an overview of the methodology used in our study. Results of the study are mentioned in Section III. Conclusions are drawn in Section IV.

\section{METHODS}

\section{A. Study}

Five T1DM adolescent patients (between the ages of 12 and 18 years old) volunteered for an overnight hypoglycemia study at the Princess Margaret Hospital for Children in Perth, Australia. The target BGL profile of the study is plotted in Fig.1. Each study consisted of five phases approximately: baseline (for reference only), euglycemia (AB), ramp (BC), hypoglycemia $(\mathrm{CD})$, and recovery (DEF). Hypoglycemia is defined as BGL lower than 3.3mmol/l.

During the study, EEG signals were continuously recorded by using a Compumedics system with the sampling rate of $128 \mathrm{~Hz}$. The EEG electrodes were positioned at four channels $\mathrm{O} 1, \mathrm{O} 2, \mathrm{C} 3$ and $\mathrm{C} 4$ according to the International 10/20 system, referenced to A1 and A2, respectively. We 


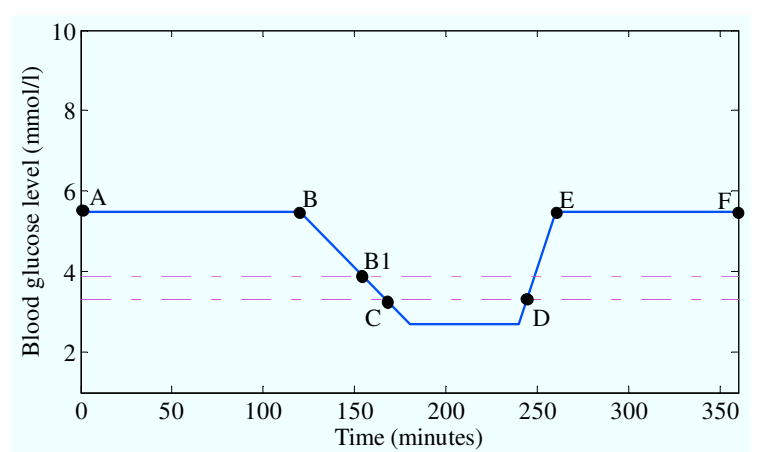

Fig. 1. Target BGL profile of the induced hypoglycemia study

also placed two electrodes at patients' chins to acquire the electro-myogram (EMG) signals and two electrodes near patients' eyes to measure the electro-oculogram (EOG) signals. The actual BGLs of patients were routinely collected to be used as reference using Yellow Spring Instruments with the general sampling period of 5 minutes. Data were collected with the approval of the Women's and Children's Health Service, Department of Health, Government of Western Australia, and with informed consent.

\section{B. Signal Processing and Feature Extraction}

After finalizing the signal acquiring step, signal processing is carried out to eliminate unwanted noise and artifact in signal. First, raw EEG data are filtered by using an IIR highpass filter with a cut-off frequency of $2 \mathrm{~Hz}$ to get rid of low frequency artifacts and a notch filter at $50 \mathrm{~Hz}$ to remove power noise. A visual artifact rejection method is applied to exclude epochs contaminated with artifacts. Segments containing significant artifacts are discarded, based on EMG and EOG signals. Finally, at each blood sampling point, a 40-second epoch of non-artifact signal is extracted. Each epoch is labeled as Normal, Early Onset, Hypoglycemia or Recovery based on the corresponding phase of the study. Referring to Fig. 1, Normal is defined as segment ABB1, Early Onset is defined as segment B1C, Hypoglycemia is defined as segment CD and Recovery is defined as segment DEF.

Spectral analysis is applied to explore EEG signals in frequency domain. Non-artifact 40-second EEG epochs are segmented into 5-second non-overlapping segments. By using Fast Fourier Transform (FFT), each segment is transformed into the frequency domain which results in the power spectrum $P\left(f_{i}\right)$, with frequency resolution of $0.2 \mathrm{~Hz}$. The power spectrum is then subdivided into 3 frequency bands: theta $(\theta: 4-8 \mathrm{~Hz})$, alpha $(\alpha: 8-13 \mathrm{~Hz})$ and beta $(\beta: 13-$ $30 \mathrm{~Hz}$ ). Previous EEG studies showed that the reduced alpha activity is associated with the lack of vigilance or awareness $[10,11]$. This is a common symptom to T1DM patients which normally happens under hypoglycemia conditions $[4$, 12]. In our previous work, it is demonstrated that there is no significant and consistent response in the beta frequency band. Because of these reasons, in this study, we concentrate on analyzing EEG spectral parameters that characterize the shift of power spectrum within theta and alpha bands. Thus, from the spectrum of each band, the following four features are estimated.
Power $(\boldsymbol{P})$ : The power level within each frequency band is a common feature in EEG research. In our study, the power level within each frequency band is estimated from the power spectrum $P\left(f_{i}\right)$ by using a numerical integration technique (the trapezoidal rule).

Centroid Frequency $(\boldsymbol{C F})$ : This feature can be referred as the center of gravity of the spectrum within each band. It is estimated as the frequency which subdivides the area under the spectral curve into identical parts.

$$
C F=\frac{\sum_{i} f_{i} P\left(f_{i}\right)}{\sum_{i} P\left(f_{i}\right)}
$$

Spectral Variance $(\mathrm{SV})$ : This feature indicates how the power spectrum is spread out within each frequency band.

$$
S V=\frac{\sum_{i} f_{i}^{2} P\left(f_{i}\right)}{\sum_{i} P\left(f_{i}\right)}-C F^{2}
$$

Spectral Entropy (SE): This feature is a measure of the distribution of normalized power spectrum within a frequency range. This feature reflects the distribution in the power spectrum. It reaches to maximum when all frequencies in the power spectrum have the same power level. In the case that power spectrum concentrates in a smaller frequency range, the SE will decrease.

$$
S E=-\frac{1}{\log N_{f}} \sum_{f} P_{n}\left(f_{i}\right) \log P_{n}\left(f_{i}\right)
$$

where $N_{f}$ is the number of frequency bins within each frequency band.

In previous work, we established that there are similarities in EEG responses between channels in the same brain areas (i.e. $\mathrm{O} 1$ and $\mathrm{O} 2$ in the occipital area; $\mathrm{C} 3$ and $\mathrm{C} 4$ in the central area). Therefore, in this paper, for the comparison purpose, we only employ data from two channels $\mathrm{C} 3$ and $\mathrm{O} 2$ which are in two different sides and areas of the brain. As a result, a total of 16 EEG features (4 different kinds of feature x 2 frequency bands x 2 channels) are estimated for each epoch. To reduce the variability in data, each final data point is estimated as the average of two consecutive nonoverlapping points.

Statistics is applied to compare and determine the significance of changes in EEG signals between three different states of Normal, Hypoglycemia and Recovery. First, descriptive analysis is carried out to assess data distribution like mean, standard deviation, normality, skewness, etc. Statistical analysis of differences between pairs of groups is performed using $t$-test for features with normal distribution and the nonparametric Wilcoxon test for features with non-normal distribution. The correlations between EEG parameters and actual BGLs during the study are also analyzed by using a nonparametric ranking test. In all tests, probability values ( $p$-values) less than 0.05 were considered to be significant. Statistical analysis is also implemented with the data from Early Onset data set which corresponds with BGL range of 3.3-3.9 $\mathrm{mmol} / \mathrm{l}$ to explore the differences of EEG responses at two BGL thresholds of 3.3 $\mathrm{mmol} / \mathrm{l}$ and $3.9 \mathrm{mmol} / \mathrm{l}$. 


\section{RESULTS}

The actual BGL profile of five patients which were collected during the study by the Yellow Spring Instruments is plotted in Fig. 2.

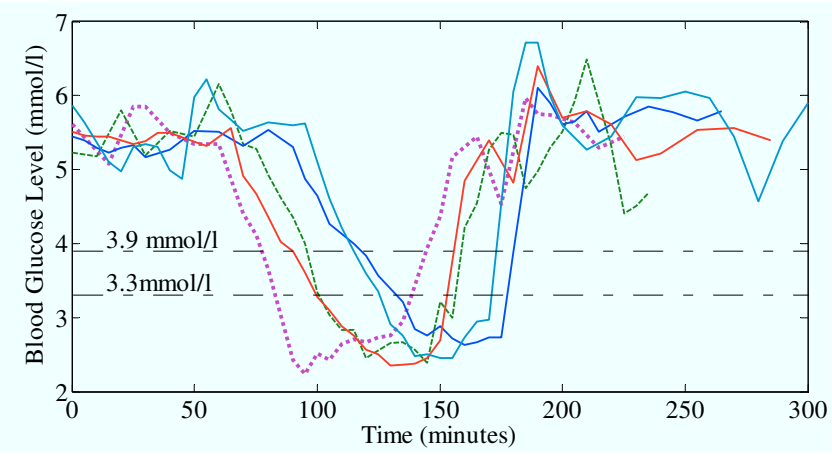

Fig.2. Actual blood glucose level profiles in 5 T1DM adolescents

Statistical results of comparison between three phases of Normal, Hypoglycemia and Recovery are presented in Table I. Significant tests are reported in bold. The centroid alpha frequency is showed to be the most significant feature which is considerably correlated to BGLs during the study at both channels $\mathrm{C} 3$ and $\mathrm{O} 2(p<0.0001)$. Group comparisons also indicate a significant decrease in the centroid alpha frequency under hypoglycemia conditions, and then a re-establishment under recovery conditions. There are significant changes in two other features of alpha bands which are spectral variance and spectral entropy at both channels. Under hypoglycemia conditions, a decrease in these two features show that the spectrum of the alpha band tends to concentrate on a narrower range. Based on the mentioned results, we establish that during hypoglycemia, in the power spectrum of alpha band, there is a shift toward smaller frequencies as showed in Fig. 3 where an example of alpha spectrums at two different states is presented.

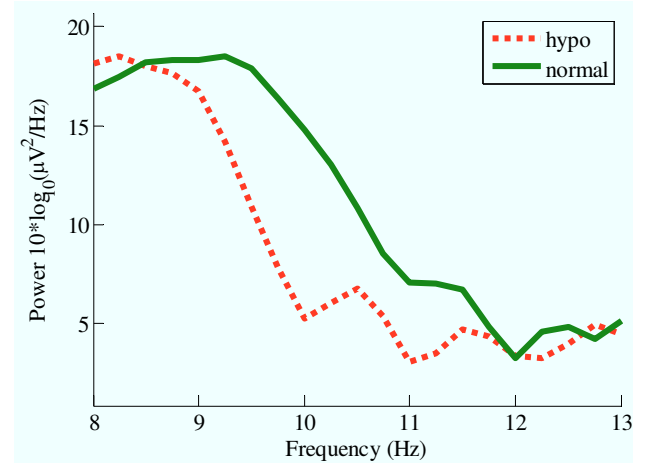

Fig.3. Example of changes in alpha spectrum

The statistical results also produce a slightly significant increase in centroid theta frequency at channel $\mathrm{O} 2(p=0.003)$. It is showed that the change in theta band is more significant at the channel $\mathrm{O} 2$ than channel $\mathrm{C} 3$. The correlation analysis produces slight changes in the power level of theta band at channel C3 $(p=0.012)$ and alpha band at channels $\mathrm{O} 2$ $(p=0.01)$. However, these changes are not consistent with the group comparison which provides no difference between pairs of groups.

Comparisons between data from Normal group and Recovery group indicate similarities in all features at both two channels. Based on these results, it is recognized that during the study, EEG responses from five patients at both channels significantly change under hypoglycemia conditions and re-establish under recovery conditions. However, it should be noted that the difference between Normal group and Hypoglycemia group is slightly stronger than the difference between Recovery group and Hypoglycemia group. These results confirm conclusions from previous studies that the occurrence of hypoglycemia can cause alternations in EEG signals and lead to impairments to the brain functioning of patients.

TABLE I. STATISTICAL RESULTS

\begin{tabular}{|c|c|c|c|c|c|c|c|c|c|}
\hline \multirow{2}{*}{\multicolumn{2}{|c|}{ Parameters }} & \multicolumn{2}{|c|}{ Correlation to BGLs } & \multicolumn{3}{|c|}{ Mean \pm SD } & \multicolumn{3}{|c|}{ Groups comparison $p$-values } \\
\hline & & $r$ & $p$-values & Normal (N) & Hypoglycemia (H) & Recovery (R) & N-H & H-R & N-R \\
\hline \multirow{8}{*}{$\mathbf{C 3}$} & $P-\theta$ & -0.098 & 0.012 & $2.105 \pm 1.328$ & $2.141 \pm 1.698$ & $1.846 \pm 1.165$ & 0.526 & 0.272 & 0.051 \\
\hline & $P-\alpha$ & -0.021 & 0.585 & $1.552 \pm 1.333$ & $1.611 \pm 1.123$ & $1.624 \pm 1.659$ & 0.202 & 0.006 & 0.143 \\
\hline & $C F-\theta$ & -0.041 & 0.294 & $5.628 \pm 0.302$ & $5.659 \pm 0.331$ & $5.610 \pm 0.290$ & 0.379 & 0.265 & 0.876 \\
\hline & $C F-\alpha$ & 0.156 & 0.001 & $10.255 \pm 0.460$ & $9.993 \pm 0.495$ & $10.132 \pm 0.510$ & $<0.001$ & 0.004 & 0.005 \\
\hline & $S V-\theta$ & -0.032 & 0.411 & $1.132 \pm 0.229$ & $1.163 \pm 0.227$ & $1.149 \pm 0.247$ & 0.208 & 0.534 & 0.562 \\
\hline & $S V-\alpha$ & 0.057 & 0.143 & $1.844 \pm 0.488$ & $1.688 \pm 0.550$ & $1.768 \pm 0.491$ & 0.002 & 0.104 & 0.099 \\
\hline & $S E-\theta$ & -0.001 & 0.987 & $0.858 \pm 0.032$ & $0.859 \pm 0.034$ & $0.860 \pm 0.038$ & 0.672 & 0.454 & 0.246 \\
\hline & $S E-\alpha$ & 0.091 & 0.019 & $0.856 \pm 0.044$ & $0.830 \pm 0.064$ & $0.847 \pm 0.051$ & $<0.0001$ & 0.013 & 0.060 \\
\hline \multirow{8}{*}{$\mathbf{O 2}$} & $P-\theta$ & -0.043 & 0.269 & $2.208 \pm 1.727$ & $2.047 \pm 1.388$ & $1.897 \pm 1.271$ & 0.908 & 0.334 & 0.259 \\
\hline & $P-\alpha$ & -0.105 & 0.010 & $2.258 \pm 2.618$ & $3.363 \pm 5.737$ & $2.644 \pm 4.212$ & 0.146 & 0.033 & 0.471 \\
\hline & $C F-\theta$ & -0.116 & 0.003 & $5.650 \pm 0.275$ & $5.754 \pm 0.377$ & $5.647 \pm 0.290$ & 0.008 & 0.011 & 0.695 \\
\hline & $C F-\alpha$ & 0.178 & $<0.0001$ & $10.133 \pm 0.392$ & $9.863 \pm 0.454$ & $10.083 \pm 0.432$ & $<0.0001$ & $<0.0001$ & 0.229 \\
\hline & $S V-\theta$ & -0.076 & 0.048 & $1.133 \pm 0.244$ & $1.196 \pm 0.247$ & $1.143 \pm 0.235$ & 0.008 & 0.042 & 0.476 \\
\hline & $S V-\alpha$ & 0.122 & 0.002 & $1.752 \pm 0.480$ & $1.553 \pm 0.576$ & $1.731 \pm 0.509$ & $<0.001$ & 0.005 & 0.329 \\
\hline & $S E-\theta$ & 0.015 & 0.703 & $0.858 \pm 0.031$ & $0.856 \pm 0.038$ & $0.859 \pm 0.033$ & 0.766 & 0.807 & 0.495 \\
\hline & $S E-\alpha$ & 0.112 & 0.005 & $0.852 \pm 0.070$ & $0.818 \pm 0.071$ & $0.850 \pm 0.048$ & $<0.001$ & $<0.001$ & 0.730 \\
\hline
\end{tabular}


The responses of four extracted EEG parameters when patients' BGLs $=3.3-3.9 \mathrm{mmol} / \mathrm{l}$ (labeled as Early Onset state) are presented in Table II. This BGL range is analyzed with the aim of investigating EEG responses at two BGL thresholds of $3.3 \mathrm{mmol} / \mathrm{l}$ and $3.9 \mathrm{mmol} / \mathrm{l}$. The results show no significant difference between the Normal state and Early Onset state except some slight changes at channel $\mathrm{O}$. Comparison between the Early Onset group and the Hypoglycemia group produces significant changes which are similar to the differences between Normal and Hypoglycemia groups. Based on these results, we establish that at the BGL area of 3.3-3.9 mmol/l, there are some slight responses in EEG parameters; however, these changes are not significant until BGL lower than $3.3 \mathrm{mmol} / \mathrm{l}$.

TABLE II. EEg RESPONSES WHEN BGL $=3.3-3.9 \mathrm{MMOL} / \mathrm{L}$

\begin{tabular}{|c|c|c|c|c|}
\hline \multirow{2}{*}{$\begin{array}{c}\text { EEG } \\
\text { parameters }\end{array}$} & \multirow{2}{*}{ Early Onset(EO) } & \multicolumn{2}{c|}{ Groups comparison $\boldsymbol{p}$-values } \\
\cline { 3 - 5 } & & N-EO & EO-H \\
\hline \multirow{4}{*}{$\mathbf{C 3}$} & $P-\theta$ & $1.960 \pm 1.464$ & 0.197 & 0.434 \\
\cline { 2 - 5 } & $P-\alpha$ & $1.509 \pm 1.062$ & 0.702 & 0.170 \\
\cline { 2 - 5 } & $C F-\theta$ & $5.632 \pm 0.267$ & 0.782 & 0.615 \\
\cline { 2 - 5 } & $C F-\alpha$ & $10.247 \pm 0.268$ & 0.904 & $<\mathbf{0 . 0 0 0 1}$ \\
\cline { 2 - 5 } & $S V-\theta$ & $1.161 \pm 0.215$ & 0.307 & $<0.0001$ \\
\cline { 2 - 5 } & $S V-\alpha$ & $1.750 \pm 0.337$ & 0.076 & 0.403 \\
\cline { 2 - 5 } & $S E-\theta$ & $0.862 \pm 0.033$ & 0.216 & 0.454 \\
\cline { 2 - 5 } & $S E-\alpha$ & $0.868 \pm 0.030$ & $\mathbf{0 . 0 3 8}$ & $<\mathbf{0 . 0 0 0 1}$ \\
\hline \multirow{4}{*}{$\mathbf{O 2}$} & $P-\theta$ & $1.943 \pm 0.877$ & 0.417 & 0.237 \\
\cline { 2 - 5 } & $P-\alpha$ & $2.344 \pm 0.980$ & 0.856 & $\mathbf{0 . 0 2 0}$ \\
\cline { 2 - 5 } & $C F-\theta$ & $5.697 \pm 0.261$ & 0.067 & $\mathbf{0 . 0 1 2}$ \\
\cline { 2 - 5 } & $C F-\alpha$ & $10.112 \pm 0.326$ & 0.851 & $<\mathbf{0 . 0 0 0 1}$ \\
\cline { 2 - 5 } & $S V-\theta$ & $1.176 \pm 0.238$ & 0.097 & 0.615 \\
\hline & $S V-\alpha$ & $1.781 \pm 0.353$ & 0.745 & $<\mathbf{0 . 0 0 1}$ \\
\cline { 2 - 5 } & $S E-\theta$ & $0.858 \pm 0.026$ & 0.544 & 0.954 \\
\hline & $S E-\alpha$ & $0.845 \pm 0.026$ & 0.068 & $\mathbf{0 . 0 2 4}$ \\
\hline
\end{tabular}

\section{CONCLUSIONS}

In this paper, four EEG spectral parameters are extracted to explore the EEG response of 5 T1DM patients during an overnight glucose clamp study. The study results indicate that under hypoglycemia conditions, there are significant changes in the alpha band of EEG signals. Under recovery conditions, these changes are shown to regain the normal state prior to hypoglycemia. However, this re-establishment does not happen consistently with all features in all patients. These results lead to a conclusion that hypoglycemia are potential to make irrecoverable damages to human brain. By analyzing the data from the BGL range of 3.3-3.9 mmol/l, we also conclude that the mentioned responses to hypoglycemia only significantly occur when patients' BGLs falling to the threshold of $3.3 \mathrm{mmol} / \mathrm{l}$.

One of the limitations of this current study is the shortage of data. The data set from five participated patients is sufficient to establish that hypoglycemia induces changes in EEG signals. However, in order to apply these results to develop a hypoglycemia detecting system that can perform in real clinical environments, other studies with more participants need to be implemented in future. Furthermore, it should be noted that this is a glucose clamp study, which is not spontaneous hypoglycemia. In future work, a natural hypoglycemia study would be carried out to explore the difference in EEG responses to insulin-induced hypoglycemia and spontaneous hypoglycemia.

With the achieved results, we are continuing to pursue our final purpose of developing the real-time system that can efficiently and continuously monitor patients' conditions and alert them and their carers when hypoglycemia is detected nocturnally during sleep.

\section{REFERENCES}

[1] U. Pedersen-Bjergaard, S. Pramming, S. R. Heller, T. M. Wallace, A. K. Rasmussen, H. V. Jørgensen, D. R. Matthews, P. Hougaard, and B. Thorsteinsson, "Severe hypoglycaemia in 1076 adult patients with type 1 diabetes: influence of risk markers and selection," Diabetes/Metabolism Research and Reviews, vol. 20, pp. 479-486, 2004.

[2] D. R. Group, "Epidemiology of severe hypoglycemia in the diabetes control and complications trial," The American journal of medicine, vol. 90, pp. 450-459, 1991.

[3] P. E. Cryer, S. N. Davis, and H. Shamoon, "Hypoglycemia in diabetes," Diabetes Care, vol. 26, pp. 1902-1912, June 1, 20032003.

[4] K. Howorka, G. Heger, A. Schabmann, P. Anderer, G. Tribl, and J. Zeitlhofer, "Severe hypoglycaemia unawareness is associated with an early decrease in vigilance during hypoglycaemia," Psychoneuroendocrinology, vol. 21, pp. 295-312, 1996.

[5] L. Hyllienmark, J. Maltez, A. Dandenell, J. Ludvigsson, and T. Brismar, "EEG abnormalities with and without relation to severe hypoglycaemia in adolescents with type 1 diabetes," Diabetologia, vol. 48, pp. 412-419, 2005.

[6] F. Laione and J. Marques, "Methodology for hypoglycaemia detection based on the processing, analysis and classification of the electroencephalogram," Medical and Biological Engineering and Computing, vol. 43, pp. 501-507, 2005.

[7] C. B. Juhl, K. Højlund, R. Elsborg, M. K. Poulsen, P. E. Selmar, J. J. Holst, C. Christiansen, and H. Beck-Nielsen, "Automated detection of hypoglycemia-induced EEG changes recorded by subcutaneous electrodes in subjects with type 1 diabetes--The brain as a biosensor," Diabetes Research and Clinical Practice, vol. 88, pp. 22-28, 2010.

[8] L. B. Nguyen, A. V. Nguyen, S. H. Ling, and H. T. Nguyen, "An adaptive strategy of classification for detecting hypoglycemia using only two EEG channels," in Engineering in Medicine and Biology Society (EMBC), 2012 Annual International Conference of the IEEE, pp. 3515-3518, 2012.

[9] L. B. Nguyen, S. S. H. Ling, T. W. Jones, and H. T. Nguyen, "Identification of hypoglycemic states for patients with T1DM using various parameters derived from EEG signals," in Engineering in Medicine and Biology Society,EMBC, 2011 Annual International Conference of the IEEE, pp. 2760-2763.

[10] E. Angelakis, J. F. Lubar, S. Stathopoulou, and J. Kounios, "Peak alpha frequency: an electroencephalographic measure of cognitive preparedness," Clinical Neurophysiology, vol. 115, pp. 887-897, Apr 2004.

[11] T. Jarm, P. Kramar, A. Zupanic, S. C. Ng, and P. Raveendran, "EEG Peak Alpha Frequency as an Indicator for Physical Fatigue," in 11th Mediterranean Conference on Medical and Biomedical Engineering and Computing 2007. vol. 16: Springer Berlin Heidelberg, 2007, pp. 517-520, 2007.

[12] K. Howorka, J. Pumprla, B. Saletu, P. Anderer, M. Krieger, and A. Schabmann, "Decrease of vigilance assessed by EEG-mapping in Type I diabetic patients with history of recurrent severe hypoglycaemia," Psychoneuroendocrinology, vol. 25, pp. 85-105, 2000. 